\title{
CÁNCER DE CUELLO UTERINO: EXPERIENCIA DURANTE UN PERÍODO DE SEIS AÑOS EN UN HOSPITAL UNIVERSITARIO COLOMBIANO
}

\author{
Cervical cancer: six years' experience in a \\ Colombian teaching hospital \\ Germán García-Soto, M.D. *; Juan de Jesús Pachón-Rincón, M.D.**; \\ Ronald Smith Meneses-Cubides, M.D.**; John Jairo Zuleta-Tobón, M.D., MsC*** \\ Recibido: junio 23/06 - Revisado: agosto 28/06 - Aceptado: enero 26/07
}

\section{RESUMEN}

Objetivo: describir las características sociodemográficas y de salud de las pacientes con cáncer invasor de cuello uterino atendidas en el Hospital Universitario San Vicente de Paúl (HUSVP) de Medellín, Colombia, durante el período 1997-2002.

Población: toda la cohorte de pacientes con diagnóstico confirmado de cáncer invasor de cuello uterino durante el período 1997-2002 en el HUSVP. Diseño: cohorte retrospectiva.

Resultados: se atendieron 735 pacientes provenientes de 11 departamentos de Colombia, principalmente de Antioquia, Chocó y Córdoba. El 20,6\% de las pacientes no tenía ningún grado de escolaridad y la mayoría era de estratos bajos. El 24,5\% de las pacientes nunca se realizó citología y solo el 20\% de los casos se diagnosticó en estadio I. El comportamiento clínico de la enfermedad fue sim1ilar a lo descrito en la literatura.

Conclusiones: en la cohorte de pacientes estudiada se evidenció un alto nivel de pobreza, bajos niveles de escolaridad, pobre cobertura de la citología cervicovaginal y un predominio notable de los estadios tardíos

\footnotetext{
* Ginecólogo oncólogo. Profesor del grupo de Ginecología Oncológica. Universidad de Antioquia. Correo electrónico: gegar65@yahoo.com.

** Residente de III año de Obstetricia y Ginecología. Universidad de Antioquia.

*** Obstetra y ginecólogo. Magíster en epidemiología clínica. Profesor del grupo de Ginecología General. Universidad de Antioquia.
}

de la enfermedad. Aunque el HUSVP es un centro que atiende personas de bajos recursos, este panorama debe llamar la atención acerca de una enfermedad tan catastrófica que se puede y se debe prevenir.

Palabras clave: cáncer de cuello uterino, citología, prevalencia, Colombia.

\section{SUMMARY}

Objective: the research was aimed at describing the health and socio-demographic characteristics of patients suffering from invasive cervical cancer who were attended at the San Vicente de Paul teaching hospital (SVPTH) in Medellin, Colombia, from 1997 to 2002.

Study design: this was a retrospective cohort study.

Population: every female patient attending SVPTH having a confirmed diagnosis of invasive cervical cancer 1997-2002 was included in the study.

Results: a total of 735 patients from 11 Colombian departments (mainly Antioquia, Chocó and Córdoba) were attended. 20.6\% of these patients had a low educational level, most coming from poor areas. 24.5\% had never sought having a Pap smear and only $20 \%$ had been diagnosed in stage I. The disease presented clinical behaviour similar to that described in previous studies. 
Conclusions: a high level of poverty, low educational level, limited Pap smear application and predominance of the disease's late stages was noticed in the cohort of patients being studied. Although SVPTH is a teaching hospital attending poor people, this scenario should call attention to a catastrophic disease which can and must be prevented.

Key words: uterine cervical neoplasms, cytology, prevalence, Colombia.

\section{INTRODUCCIÓN}

El cáncer de cuello uterino ocupa el segundo tumor en frecuencia de los cánceres de las mujeres en el mundo y constituye la primera causa de muerte por cáncer en la población femenina en los países en desarrollo. ${ }^{1,2}$ De los cerca de 500.000 casos nuevos que se presentan cada año, alrededor del $80 \%$ ocurren en estos países. ${ }^{3}$ La implementación de programas adecuados de tamización con citología cervical en los países desarrollados ha disminuido la incidencia y la mortalidad en cerca de un 75\%. ${ }^{1,4-6}$ El panorama es distinto en los países pobres donde los programas de tamización no se han implementado o han sido inadecuados y la incidencia y mortalidad siguen siendo un problema de primer orden.,

En Colombia, según datos de Globocan del año 2002, ${ }^{7}$ el cáncer de cuello uterino es el más común entre las neoplasias que afectan a la mujer. El tumor maligno más frecuentemente diagnosticado en el Instituto Nacional de Cancerología (INC) de Bogotá durante el año 2002 con 720 casos fue el cáncer de cérvix, que representó el 23,4\% de todos los cánceres diagnosticados en mujeres. ${ }^{8}$ Aunque no existen datos globales del país, la incidencia por 100.000 mujeres año según el registro poblacional de Cali era de alrededor de 34 para el año $1992^{3}$ y una cifra similar es reportada por Globocan para el año 2002. ${ }^{7}$

En el departamento de Antioquia, según el informe de su Dirección Seccional de Salud a partir del registro poblacional de cáncer, durante el año 2005 se diagnosticaron 2.757 casos de cáncer invasor de cuello uterino, representando el 16,5\% de todos los cánceres, con lo que ocupa el primer lugar en el departamento. Para este año la mortalidad por cáncer de cérvix fue de 7,3 por 100.000 mujeres. ${ }^{9}$ Pinilla y col. también habían destacado la alta incidencia de esta neoplasia en el departamento. ${ }^{10}$

Después de realizar una búsqueda manual de las revistas nacionales, se encontró que la información con respecto a las características de las mujeres que presentan cáncer de cérvix en el departamento es escasa. Esta información es necesaria tanto para las instituciones que prestan servicios a estas pacientes como para los entes encargados de planificar las políticas de salud, por lo tanto se realizó un estudio con el objetivo de describir las características sociodemográficas y de salud de las pacientes con cáncer invasor de cuello uterino atendidas en el Hospital Universitario San Vicente de Paúl (HUSVP) de Medellín, durante el período 1997-2002.

\section{MATERIALES Y MÉTODOS}

Diseño: cohorte retrospectiva de pacientes atendidas en el período comprendido entre el $1^{\circ} \mathrm{de}$ enero de 1997 y el 31 de diciembre del 2002.

Se incluyeron pacientes con diagnóstico histológico de cáncer invasor de cuello uterino atendidas en el servicio de oncología ginecológica del HUSVP. Se excluyeron mujeres que habían recibido el tratamiento inicial en otra institución y habían sido remitidas para terapia complementaria (radioterapia) o para los controles postratamiento.

El HUSVP es un centro de referencia ubicado en la ciudad de Medellín (Colombia), que atiende pacientes provenientes de la región centro oriental del país, tanto afiliados al Sistema General de Seguridad Social (régimen subsidiado y contributivo) como no afiliados (población pobre no asegurada o vinculados).

Procedimiento: se hizo búsqueda de los números de las historias clínicas de las pacientes en los registros sistematizados de la institución teniendo en cuenta los diagnósticos de ingreso y egreso hospitalario, los registros de cirugía y radioterapia 
y los registros de consulta externa. La información se obtuvo directamente de la historia clínica y se consignó en un formato estandarizado específicamente diseñado para recolectar la información de la investigación.

Variables de estudio: la edad se consideró en años cumplidos al momento del tratamiento; para la raza se tuvo en cuenta la asignada por el médico tratante; el estado civil, la escolaridad, la procedencia, la edad de la menarquia y de la menopausia y el estrato socioeconómico, según la información dada por la paciente al momento del ingreso. La escolaridad se discriminó por nivel en primaria, secundaria, universidad. Para el estrato socioeconómico se consideró cero la población indigente. En cuanto a sintomatología, se consideró sangrado la presencia de sinusorragia, flujo sanguinolento o sangrado franco. Dolor pélvico incluye dispareunia y dolor pélvico intermitente o continuo de cualquier intensidad. La clasificación clínica del estadio la realiza rutinariamente el comité de oncología ginecológica de la institución conformado por dos o tres ginecólogos oncólogos. El diagnóstico histopatológico está a cargo de los patólogos de la institución y de la Universidad de Antioquia y en la historia clínica permanece una copia del reporte. Se consideró tabaquismo el consumo de cualquier cantidad de cigarrillos por día.

Aspectos éticos: el estudio fue aprobado por el comité de ética del HUSVP y respeta la confidencialidad de las pacientes.

Análisis estadístico: las variables cualitativas se presentan en número absoluto y proporción y las cuantitativas se resumieron con mediana o promedio con su respectiva medida de dispersión, según el nivel de medición y la distribución de las mismas. Se empleó el programa SPSS ${ }^{\circledR} 13.0$ para realizar los cálculos.

\section{RESULTADOS}

En la búsqueda inicial a partir de los registros se detectaron 843 casos con diagnóstico de cáncer de cérvix. Al revisar las historias se encontró que 29 no tenían ningún diagnóstico oncológico, 21 tenían un diagnóstico oncológico diferente al de cáncer de cérvix y 34 tenían lesiones preinvasivas. Durante el período descrito se atendieron 759 pacientes con diagnóstico de cáncer de cuello uterino, 21 historias no se encontraron en el archivo luego de 3 solicitudes y 3 habían sido tratadas en otras instituciones, por tanto se analizaron 735 registros.

\section{Aspectos sociodemográficos y factores de riesgo}

La tabla 1 muestra las principales características sociodemográficas y la figura 1, la distribución por edad. En el 64,8\% de los casos no se encontró información con respecto al empleo de anticoncepción y solo el 15,1\% de las mujeres, con cuya información se contó, reportó el uso del condón por parte de sus compañeros. El 39,1\% de las 435 pacientes de quienes se obtuvo información con respecto al consumo de cigarrillo eran fumadoras. 137 pacientes $(18,5 \%)$ reconocieron que nunca se habían realizado citología y 349 (47,5\%) se habían hecho únicamente una. De las 240 pacientes en estadio III, 87 (36,3\%) nunca habían realizado este estudio, y de las 10 en estadio IV, 4 (40\%) estaban en igual condición.

Las tablas 2 y 3 muestran el porcentaje de pacientes en cada estadio de acuerdo con el estrato socioeconómico y el nivel de educación respectivamente.

\section{Aspectos clínicos de la enfermedad}

El 66,7\% de las pacientes consultó por sangrado anormal por la vagina como el síntoma principal. El 19,6\% registraron como el motivo principal de consulta la citología anormal y el 3,7\% manifestó dolor pélvico como primer síntoma. En el 8,6\% de los casos no se reportó cuál fue el motivo inicial de consulta.

La figura 2 muestra el estadio de la enfermedad de 719 pacientes, según el sistema de clasificación de la Federación Internacional de Ginecología y Obstetricia (FIGO). 16 historias clínicas no tienen la clasificación del estadio. 


\section{Tabla 1. Características sociodemográficas de las pacientes con cáncer invasor de cuello ute- rino atendidas en el HUSVP durante el período 1997-2002}

\begin{tabular}{|l|c|}
\hline Edad, años media (ds) & $48,3(13)$ \\
\hline $\begin{array}{l}\text { Edad de la menarca, } \\
\text { años media (ds) }\end{array}$ & $13(1,45)$ \\
\hline $\begin{array}{l}\text { Edad de la menopausia, } \\
\text { años media (ds) }\end{array}$ & $47,9(4,8)$ \\
\hline $\begin{array}{l}\text { Edad de inicio de la vida sexual, } \\
\text { años media (ds) }\end{array}$ & $16(3,3)$ \\
\hline $\begin{array}{l}\text { Embarazos, mediana (p 25-75) } \\
\text { Abortos, mediana (p 25-75) }\end{array}$ & $6(3-9)$ \\
\hline $\begin{array}{l}\text { Número de compañeros sexuales, } \\
\text { mediana (p 25-75) }\end{array}$ & $2(1-3)$ \\
\hline Tabaquismo (435/735)*n (\%) & $170(23,1)$ \\
\hline Realización de citología \\
$(560 / 735) *$
\end{tabular}

* Número de pacientes con información/total de pacientes.

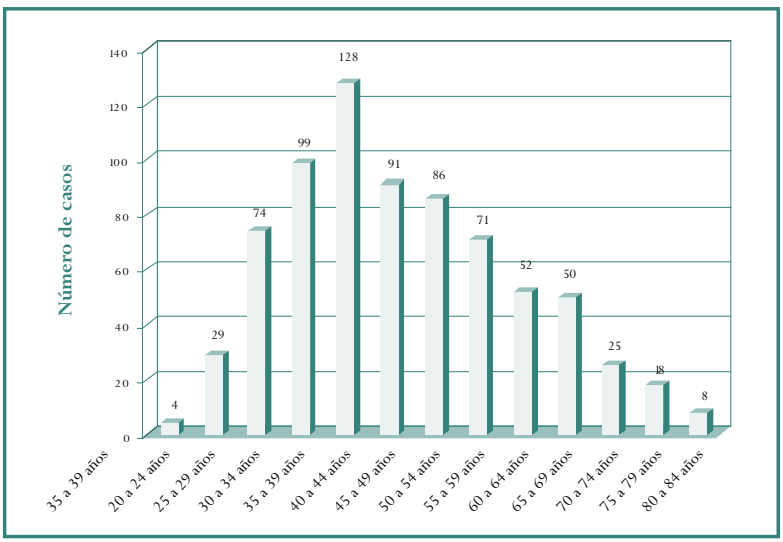

Figura 1. Número de casos según grupos de edad de 735 pacientes con cáncer invasor de cuello uterino atendidas en el HUSVP durante el período 1997-2002

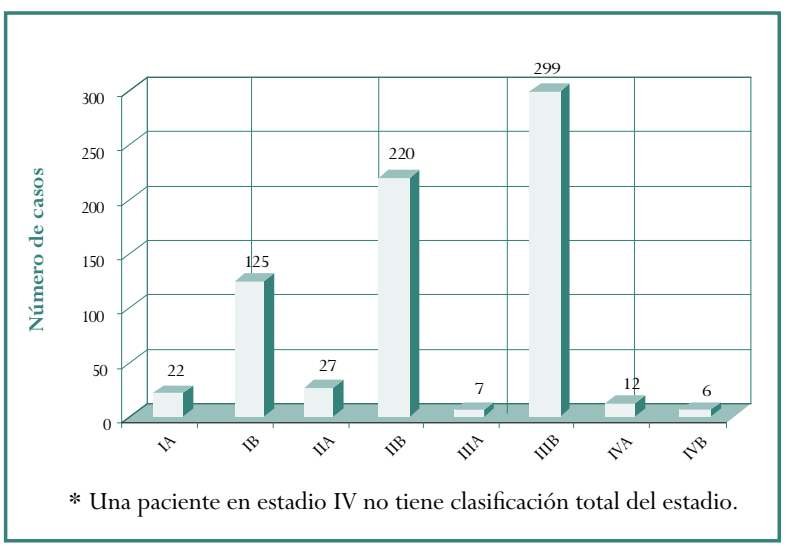

Figura 2. Estadio FIGO de 719 pacientes con cáncer invasor de cuello uterino atendidas en el HUSVP durante el período 1997-2002

El tipo histológico predominante fue el escamocelular con el 88,6\% de los casos, el adenocarcinoma representó el 10,6\% de los casos y el 0,8\% fueron del tipo adenoescamoso y otros menos frecuentes.

\section{Aspectos relacionados con el tratamiento}

La tabla 4 muestra el número y porcentaje de pacientes en cada uno de los esquemas de tratamiento según el estadio. Las 16 pacientes de las que no se consignó el estadio en la historia fueron tratadas así: 8 con radioterapia, 3 con combinación de radio y quimioterapia, 2 con cirugía, 1 con quimioterapia, 1 no recibió ningún tratamiento y otra no tiene información. 
Tabla 2. Proporción de pacientes por estadio distribuido según el estrato socioeconómico

\begin{tabular}{|c|c|c|c|c|c|c|}
\hline \multirow{2}{*}{ Estadio } & \multicolumn{5}{|c|}{ Estrato } & \multirow{2}{*}{$\begin{array}{l}\text { Total casos } \\
\text { por estadio }\end{array}$} \\
\hline & 0 & 1 & 2 & 3 & 4 & \\
\hline I & $14,3 \%$ & $15 \%$ & $21,6 \%$ & $40,7 \%$ & $0 \%$ & 105 \\
\hline II & $35,7 \%$ & $34,3 \%$ & $35,8 \%$ & $18,5 \%$ & $100 \%$ & 192 \\
\hline III & $50 \%$ & $47,7 \%$ & $41,3 \%$ & $33,3 \%$ & $0 \%$ & 249 \\
\hline IV & $0 \%$ & $3 \%$ & $1,4 \%$ & $7,4 \%$ & $0 \%$ & 14 \\
\hline $\begin{array}{l}\text { Total casos } \\
\text { por estrato }\end{array}$ & $14(100 \%)$ & $300(100 \%)$ & 218 (100\%) & 27 (100\%) & $1(100 \%)$ & $560 *$ \\
\hline
\end{tabular}

* Pacientes para las que se cuenta simultáneamente con información del estrato y del estadio.

Tabla 3. Proporción de pacientes por estadio distribuido según el nivel educativo

\begin{tabular}{|l|c|c|c|c|c|}
\hline \multicolumn{1}{|c|}{ Estadio } & Ninguno & Primaria & Secundaria & Universitario & $\begin{array}{c}\text { Total por } \\
\text { estadio }\end{array}$ \\
\hline I & $12,8 \%$ & $20 \%$ & $33,3 \%$ & $50 \%$ & 112 \\
\hline II & $29,1 \%$ & $38,4 \%$ & $36,8 \%$ & $50 \%$ & 203 \\
\hline III & $57,3 \%$ & $40,5 \%$ & $29,8 \%$ & $0 \%$ & 238 \\
\hline IV & $0,9 \%$ & $1,1 \%$ & $0 \%$ & $0 \%$ & 5 \\
\hline $\begin{array}{l}\text { Total casos } \\
\text { por nivel }\end{array}$ & $\mathbf{1 1 7}$ & $\mathbf{3 8 0}$ & $\mathbf{5 7}$ & $\mathbf{4}$ & $\mathbf{5 5 8 *}$ \\
\hline
\end{tabular}

* Pacientes para las que se cuenta simultáneamente con información del nivel educativo y del estadio.

Tabla 4. Tipo de tratamiento recibido por las pacientes con cáncer invasor de cuello uterino atendidas en el HUSVP durante el período 1997-2002

\begin{tabular}{|c|c|c|c|c|c|}
\hline \multirow{2}{*}{ Tratamiento } & & & & & \multirow{2}{*}{$\begin{array}{l}\text { Total por } \\
\text { tratamiento }\end{array}$} \\
\hline & I & II & III & IV & \\
\hline Ninguno* & $1(0,7 \%)$ & $1(0,4 \%)$ & $24(7,8 \%)$ & $6(33,3 \%)$ & $32(4,5 \%)$ \\
\hline Cirugía** & $79(53,7 \%)$ & $4(1,6 \%)$ & $0(0 \%)$ & $0(0 \%)$ & $83(11,6 \%)$ \\
\hline Radioterapia*** & $36(24,5 \%)$ & $227(91,9 \%)$ & $267(87,3 \%)$ & $12(61,1 \%)$ & $541(75,3 \%)$ \\
\hline Quimioterapia & $0(0 \%)$ & $4(1,6 \%)$ & $12(3,9 \%)$ & $1(5,6 \%)$ & $17(2,4 \%)$ \\
\hline Radio y quimioterapia & $31(21,1 \%)$ & $9(3,6 \%)$ & $1(0,3 \%)$ & $0(0 \%)$ & $41(5,7 \%)$ \\
\hline Sin información & $0(0 \%)$ & $2(0,8 \%)$ & $2(0,7)$ & $0(0 \%)$ & $4(0,6 \%)$ \\
\hline Total por estadio & 147 & 247 & 306 & 19 & 718 \\
\hline
\end{tabular}

* Pacientes que rehusaron cualquier tratamiento o murieron antes de ser tratadas.

** Histerectomía radical más linfadenectomía pélvica.

*** Radioterapia externa más braquiterapia.

En dos casos el porcentaje final no suma exactamente el 100\% por efectos de la aproximación. 


\section{Aspectos relacionados con las complicaciones del tratamiento}

La hemorragia se presentó solamente en 3 pacientes $(2,3 \%)$ de todas las que fueron sometidas a cirugía.

Las complicaciones reportadas con más frecuencia en la historia fueron la proctitis actínica $(13,6 \%)$, las fístulas $(2,7 \%)$ y la cistitis actínica $(1,1 \%)$, sin embargo no se cuenta con información del 17,8\% de las pacientes.

\section{Aspectos relacionados con la supervivencia y el estado final}

$172(23,4 \%)$ no tienen seguimiento en la institución después de haber recibido el tratamiento, el 50\% tienen un seguimiento inferior a 9 meses y el 75\% inferior a 28 meses.

Existe documentación de muerte de 57 (7,8\%), todas relacionadas con la enfermedad: 5 de estadio IB, 8 del IIB, 35 del IIIB, 5 del IVA y 2 del IVB.

\section{DISCUSIÓN}

Aunque la población que se atiende en el HUSVP ya ha sido en parte seleccionada por efectos del sistema de contratación, el panorama general visto a través de los resultados de este estudio es que las mujeres que sufren de cáncer de cérvix invasor son una población con un perfil de altos niveles de pobreza, bajos niveles de escolaridad, muy baja cobertura en la realización de citologías en esquema adecuado y por consiguiente de alta incidencia de estadios avanzados de la enfermedad. Esto está en concordancia con lo reportado en la literatura para los países en desarrollo. ${ }^{7,11}$

En la cohorte de estudio se encontró un número importante de casos en mujeres menores de 30 años de edad. Este aspecto lo habían reseñado Gallego y cols. en un estudio previo, ${ }^{12}$ y se puede atribuir, por lo menos en parte, al inicio temprano de la actividad sexual encontrado. Igualmente se hallaron casos en mujeres mayores de 70 y 80 años, lo cual se debe tener en cuenta al momento de extrapolar ${ }^{13}$ recomendaciones de otros médicos de suspender la tamización después de los $65,{ }^{14}$ pues se debe considerar la población local como de riesgo incluso antes y después de estos límites. Se encontró un promedio alto de embarazos, aspecto que algunos han señalado como un factor de riesgo para cáncer de cuello uterino. ${ }^{4}$

La no realización de la citología, la demora para consultar ante los primeros síntomas y el tener estadios avanzados de la enfermedad se notó preponderantemente entre las mujeres más pobres, las de menor nivel educativo y entre las de mayor edad. Se registró que un 63\% de las pacientes se realizó al menos una citología, pero esta pudo haber correspondido a la solicitada por el médico ante algún síntoma que finalmente llevó al diagnóstico; de tal manera que muy probablemente muchas no están dentro de los programas de la tamización. Sin embargo, no fue posible aclarar este aspecto.

Globalmente, el 76\% de las pacientes se diagnosticó en estadios tardíos (IIB-IVB). Otros informes de poblaciones colombianas muestran una tendencia similar, como el del INC de Bogotá, donde en el año 2002 el 49,1\% de los cánceres invasores del cuello uterino se encontraron en estadio IIIB. ${ }^{7}$

El esquema combinado de radioterapia y quimioterapia se usó solamente en el 2,4\% de las pacientes. Este esquema se comenzó a usar en el HUSVP hacia finales del año 2000, luego de la aparición de estudios que daban cuenta de su mayor eficacia frente al esquema de radioterapia sola. ${ }^{15}$ El componente de quimioterapia se basó en Platino, de acuerdo con los esquemas aceptados. ${ }^{16}$ El tratamiento según el estadio es similar a lo reportado en la literatura. Las muertes documentadas se presentaron predominantemente en estadios avanzados, que son el mayor determinante del pronóstico. ${ }^{17,18}$

Las complicaciones vinculadas con el tratamiento se relacionan principalmente con la modalidad terapéutica. ${ }^{15,19}$ Las lesiones actínicas fueron las más numerosas, acorde con el gran porcentaje de pacientes que recibieron radioterapia por estar en estadios avanzados de la enfermedad.

El seguimiento adecuado de las pacientes tratadas por cáncer de cuello uterino es un componente fundamental de la atención y este estudio encontró que este aspecto es crítico en la institución, situación que 
posiblemente no es ajena a otras instituciones locales. Una de las explicaciones encontradas para esto es el contexto actual del sistema de salud colombiano: no existe continuidad en la atención y las pacientes deben continuar sus controles en sitios diferentes a los que hicieron la atención inicial de la enfermedad. Esta ausencia de información impide evaluar la efectividad de los tratamientos administrados, dificulta conocer la supervivencia y hace imposible saber el efecto que se logra con la modificación de los esquemas terapéuticos, que aunque se hayan probado en otros medios, es importante por sus costos conocer si realmente ofrecen beneficios dentro de la realidad del país, como es la reciente adición de la quimioterapia a las pacientes tributarias de radioterapia.

Los resultados de esta investigación deben motivar a los diversos sectores del sistema de salud de Antioquia y de otras regiones del país para un cambio de actitud frente a esta enfermedad catastrófica que se puede y se debe prevenir.

\section{REFERENCIAS}

1. Waxman AG. Guidelines for cervical cancer screening: history and scientific rationale. Clin Obstet Gynecol 2005;48:77-97.

2. Borrero M. Actualización en citología cervicovaginal. En: XI Curso de Actualización en Obstetricia y Ginecología. Universidad de Antioquia; 2003. p. 41-50.

3. Waggoner SE. Cervical cancer. Lancet 2003;361: 2217-25.

4. Morris M, Tortolero-Luna G, Malpica A. Neoplasia intraepitelial y cáncer cervicouterino. En: Clínicas de ginecología y obstetricia. Temas actuales. McGraw Hill Interamericana; 1996, Vol. 2. p. 315-73.

5. Krivac T, Mcbroom J. Cáncer cervicouterino y vaginal. En: Berek J. Ginecología de Novak. McGraw Hill Interamericana. 13ª edición; 2003. p. 959-87.

6. Lazcano-Ponce E, Alonso P, Ruiz-Moreno JA, Hernandez-Avila M. Recommendations for cervical cancer screening programs in developing countries. The need for equity and technological development. Salud Publica Mex 2003;45 Suppl 3:S449-62.
7. Globocan 2002 database. Cancer incidence, mortality and prevalence worldwide. http://www.dep-iarc.fr/

8. Pardo C, Murillo R, Piñeros M, Castro MA. Casos nuevos de cáncer en el Instituto Nacional de Cancerología, Colombia, 2002. Rev Colomb Cancerol 2003;7:4-19.

9. Dirección Seccional de Salud de Antioquia. Registro poblacional de cáncer. [Visitado 2007, enero] Disponible en: http://www.dssa.gov.co/dowload/ CANCER2005.pdf

10. Pinilla E, Uribe J, González G. Cáncer de cérvix IB. Análisis de 110 casos. Rev Colomb Obstet Ginecol 1988;39:186-90.

11. Arrossi S, Sankaranarayanan R, Parkin DM. Incidence and mortality of cervical cancer in Latin America. Salud Publica Mex 2003;45 Suppl 3:S306-14.

12. Gallego G, Uribe J, Ruiz IC. Carcinoma de cérvix en mujeres menores de 25 años. IATREIA1993;6:61-6.

13. Ministerio de Salud. Dirección General de Promoción y Prevención. Norma técnica para la detección temprana del cáncer de cuello uterino y guía de atención de lesiones preneoplásicas de cuello uterino. 2000.

14. ACOG Committee on Practice Bulletins. ACOG Practice Bulletin: clinical management guidelines for obstetriciangynecologists. Number 45, August 2003. Cervical cytology screening (replaces committee opinion 152, March 1995). Obstet Gynecol 2003;102:417-27.

15. Morris M, Eifel PJ, Lu J, Grigsby PW, Levenback C, Stevens RE, et al. Pelvic radiation with concurrent chemotherapy compared with pelvic and para-aortic radiation for high-risk cervical cancer. $N$ Engl J Med 1999;340:1137-43.

16. Rose PG, Bundy BN, Watkins EB, Thigpen JT, Deppe G, Maiman MA, et al. Concurrent cisplatin-based radiotherapy and chemotherapy for locally advanced cervical cancer. N Engl J Med 1999;340:1144-53.

17. Holtz DO, Dunton C. Tratamiento actual del cáncer cervicouterino invasor. En: Prevención de la mortalidad y morbilidad por cáncer cervicouterino. Clínicas de Ginecología y Obstetricia. Temas actuales. McGraw Hill Interamericana. Vol. 4, 2002. p. 645-56.

18. Hacker NF. Cervical cancer. En: Berek JS, Hacker NF. Practical gynecologic oncology. 3rd ed. Philadelphia: Lippincott Williams \& Wilkins; 2000. p. 380.

19. Eifel P. Radiation therapy. En: Practical gynecologic oncology, 3rd ed. Philadelphia: Lippincott Williams \& Wilkins; 2000. p. 146. 